\title{
Epsilon expansion for infrared Yang-Mills theory in Landau gauge
}

\author{
Axel Weber \\ Instituto de Física y Matemáticas, Universidad Michoacana de San Nicolás de Hidalgo, \\ Edificio C-3, Ciudad Universitaria, \\ A. Postal 2-82, 58040 Morelia, Michoacán, Mexico
}

(Dated: November 19, 2018)

\begin{abstract}
The study of the Dyson-Schwinger equations of Landau gauge Yang-Mills theory has revealed two types of solutions for the gluon and ghost propagators, with a scaling and a massive (decoupling) behavior in the extreme infrared, respectively. We show that both types of solutions are quantitatively reproduced by applying renormalization group equations of Callan-Symanzik type in an epsilon expansion to the infrared limit of Landau gauge Yang-Mills theory when a mass term for the gluons is added to the action. Only the decoupling solution corresponds to an infrared-stable fixed point in three and four space-time dimensions and is hence expected to be physically realized, in agreement with the results of recent lattice calculations.
\end{abstract}

PACS numbers: 12.38.Aw, 12.38.Lg, 11.10.Jj

Keywords: Yang-Mills theory, infrared propagators, renormalization group, epsilon expansion 
The infrared (IR) behavior of gluon and ghost propagators in $S U(N)$ Yang-Mills theory in Landau gauge has been a subject of intense debate over the last decade. The first results that have been obtained in the extreme IR region, for momentum scales $p \ll \Lambda_{\mathrm{QCD}}$, came from the solution of an approximation to the full Dyson-Schwinger (DS) equations in this theory [1]. They are nowadays known as scaling solutions, showing power-law behavior of the propagators in the IR.

For a quantitative description of these results, we introduce some notation. The gluon and ghost propagators in $D$ Euclidean space-time dimensions are parameterized as

$$
\begin{aligned}
\left\langle A_{\mu}^{a}(p) A_{\nu}^{b}(-q)\right\rangle & =\frac{G\left(p^{2}\right)}{p^{2}}\left(\delta_{\mu \nu}-\frac{p_{\mu} p_{\nu}}{p^{2}}\right) \delta^{a b}(2 \pi)^{D} \delta(p-q), \\
\left\langle c^{a}(p) \bar{c}^{b}(-q)\right\rangle & =\frac{F\left(p^{2}\right)}{p^{2}} \delta^{a b}(2 \pi)^{D} \delta(p-q) .
\end{aligned}
$$

The scaling solutions show a power behavior of the dressing functions in the IR,

$$
G\left(p^{2}\right) \propto\left(p^{2}\right)^{-\alpha_{G}}, \quad F\left(p^{2}\right) \propto\left(p^{2}\right)^{-\alpha_{F}}
$$

The anomalous dimensions $\alpha_{G}, \alpha_{F}$ fulfill a sum rule,

$$
\alpha_{G}+2 \alpha_{F}=\frac{D-4}{2}
$$

The approximate DS equations can be solved analytically in the IR regime [2, 3]. There are two consistent solutions, one of them giving

$$
\alpha_{F}(D)=\frac{D-2}{2}, \quad \alpha_{G}(D)=-\frac{D}{2},
$$

for $2<D<4$. The IR exponents of the other solution are obtained, for $2 \leq D \leq 4$, in general numerically from a transcendental equation. However, a very good approximation (within $2 \%$ error) is given by

$$
\alpha_{F}(D)=\frac{D-1}{5}, \quad \alpha_{G}(D)=-\frac{16-D}{10} .
$$

Note that, within the range of dimensions indicated, for both solutions $\alpha_{F}>0$ and $\alpha_{G}<-1$ hold, which means that the ghost propagator is enhanced in the IR with respect to the treelevel propagator, while the gluon propagator tends to zero for $p \rightarrow 0$. As a consequence, in the IR the diagrams with the greatest number of ghost propagators dominate over the others, a property called ghost dominance. 
Of particular interest is the IR behavior of a dimensionless running coupling constant defined from the ghost-gluon vertex. Taylor's well-known argument [4] implies that in the Landau gauge at the symmetrical point the proper ghost-gluon vertex receives only finite and scale-independent quantum corrections, to all perturbative orders. This fact motivates the definition

$$
g_{R}^{2}\left(p^{2}\right)=\left(p^{2}\right)^{(D-4) / 2} G\left(p^{2}\right) F^{2}\left(p^{2}\right) g^{2}
$$

of the dimensionless renormalized coupling constant $g_{R}$ in terms of the bare coupling constant $g$. As a consequence of the sum rule (3), for the scaling solutions $g_{R}$ tends toward a nonzero constant in the limit $p \rightarrow 0$. The value of $g_{R}(0)$ can be obtained from the IR solutions of the DS equations.

Several years after the discovery of the scaling solutions, however, another type of solutions of the same approximate DS equations have been found, called decoupling solutions [5 7]. The latter solutions are characterized by $\alpha_{F}=0$ and $\alpha_{G}=-1$, for any dimension $D$ in the range $2<D \leq 4$. In particular, for these solutions the sum rule (3) does not hold. Qualitatively, in the decoupling solutions the ghost propagator is enhanced in the IR at most by a finite factor, while the gluon propagator tends towards a nonzero constant for $p \rightarrow 0$, similar to the propagator in a massive theory. Consequently, the dimensionless running coupling constant defined in Eq. (6) vanishes in the IR limit, contrary to the scaling solutions.

Ever since the scaling solutions have been found, lattice calculations in the Landau gauge have focused on the extreme IR regime. The subject has been controversial over many years, but as for today there is quite convincing evidence that these numerical simulations find gluon and ghost propagators of the decoupling type in dimensions $D=3,4[8-10]$ and scaling solutions in $D=2[11]$.

In the present paper, we show that a renormalization group (RG) analysis in an epsilon expansion is capable of quantitatively reproducing both the scaling and the decoupling solutions in the extreme IR, and furthermore of determining the IR-stability of the solutions. Only solutions corresponding to IR-stable fixed points are expected to be physically realized. Of course, it is well known that in the standard formulation of the theory the only perturbatively accesible fixed point is the trivial one. It is UV-attractive and IR-repulsive and can therefore not be used for a description of the IR physics. We here introduce a minimal modification of standard Yang-Mills theory by adding a mass term for the gluons 
as in Ref. [12], see also [13]. The gluon mass is generically expected to be of the order of the QCD scale $\Lambda_{\mathrm{QCD}}$. It is well known that this modification does not affect the UV behavior of the theory. In particular, the beta function in this aymptotic region is unchanged and asymptotic freedom is maintained. A gluon mass term has been considered in many contexts before, often on phenomenological grounds.

Our main motivation for the introduction of a gluon mass term, however, comes from new theoretical results: the quantization of Yang-Mills theory in the Landau gauge is complicated by the existence of gauge copies, and the corresponding restriction of the functional integral over the gauge fields to the first Gribov region breaks the BRST invariance of the effective action [14, 15]. From the RG viewpoint, the appearance of a gluon mass term is a natural consequence of the breaking of BRST invariance. Indeed, it has been shown in Ref. [15] that a gluon mass term arises as a result of the formation of condensates of auxiliary fields that are introduced in order to implement the restriction to the Gribov region by adding local operators to the action. In the present analysis, we will not take any additional auxiliary fields into account.

In the following, we will hence consider the $D$-dimensional Euclidean action

$$
S=\int d^{D} x\left(\frac{1}{4} F_{\mu \nu}^{a} F_{\mu \nu}^{a}+\frac{1}{2} A_{\mu}^{a} m^{2} A_{\mu}^{a}+\partial_{\mu} \bar{c}^{a} D_{\mu}^{a b} c^{b}+i B^{a} \partial_{\mu} A_{\mu}^{a}\right)
$$

with the gluon mass $m$, as the classical action for $S U(N)$ Yang-Mills theory in the Landau gauge. The Nakanishi-Lautrup field $B^{a}$ is used to conveniently implement the restriction to transverse gauge fields, $\partial_{\mu} A_{\mu}^{a}=0$. As we shall now argue, the existence of a gluon mass term profoundly changes the RG analysis of the theory in the IR.

Naively, for momenta $p$ with $p^{2} \ll m^{2}$, the mass term in Eq. (7) dominates the contributions to the action that are quadratic in the gluon field. In fact, this is also true for the loop corrections. As a relevant example, consider the one-loop contribution to the ghost self-energy in dimensions $2 \leq D \leq 4$ : it is straightforward to show that one may replace the tree-level gluon propagator corresponding to the action (7) inside the loop diagram with

$$
\frac{1}{m^{2}}\left(\delta_{\mu \nu}-\frac{p_{\mu} p_{\nu}}{p^{2}}\right) \delta^{a b}(2 \pi)^{D} \delta(p-q)
$$

and obtain exactly the same result in the IR limit, with corrections suppressed by powers of $p^{2} / m^{2}$, after renormalization with an appropriate normalization condition for the term $\propto p^{2}$ in the ghost two-point function. For the description of the extreme IR regime, we will 
hence in the following reduce the complete term quadratic in the gluon field in the action (7) to the mass term.

We initiate the RG analysis by considering the limit $g \rightarrow 0$ of the approximate IR-form of the action which then reduces to

$$
S_{\mathrm{IR}}^{0}=\int d^{D} x\left(\frac{1}{2} A_{\mu}^{a} m^{2} A_{\mu}^{a}+\partial_{\mu} \bar{c}^{a} \partial_{\mu} c^{a}+i B^{a} \partial_{\mu} A_{\mu}^{a}\right) .
$$

Under a rescaling of the space-time coordinate $x \rightarrow x / s$ with $s>1$ [to be understood as one step in a Wilsonian RG transformation for the free field theory defined by the action (9)] ], the IR-action is invariant provided that the fields transform as

$$
\begin{array}{rlrl}
A_{\mu}^{a}(x) & \rightarrow s^{D / 2} A_{\mu}^{a}(s x), & B^{a}(x) & \rightarrow s^{(D / 2)-1} B^{a}(s x), \\
c^{a}(x) & \rightarrow s^{(D / 2)-1} c^{a}(s x), & \bar{c}^{a}(x) \rightarrow s^{(D / 2)-1} \bar{c}^{a}(s x)
\end{array}
$$

( $m^{2}$ being constant under the rescaling). Note that Eq. (10) now defines the canonical or scaling (mass) dimensions of the fields which differ, in the case of the gluon field and the Nakanishi-Lautrup field, from the usual canonical dimensions which refer to the Gaussian fixed point. To reconcile the scaling dimension of $A_{\mu}^{a}$ with the usual canonical dimension, we may absorb one power of the mass in the gluon field to define

$$
\tilde{A}_{\mu}^{a}(x)=m A_{\mu}^{a}(x)
$$

and consider $\tilde{A}_{\mu}^{a}$ as the fundamental field. Similarly, we define $\tilde{B}^{a}(x)=B^{a}(x) / m$. As far as the gluon field is concerned, the fixed point action (9) corresponds to the high-temperature fixed point rather than the usual Gaussian fixed point [16]. It is natural to associate the high-temperature fixed point with a stochastic vacuum state.

We will now reintroduce the coupling terms appearing in the Yang-Mills action. An important consequence of the scaling dimensions (10) is that the ghost-gluon, three-gluon, and four-gluon couplings all receive different scaling dimensions:

$$
g_{\bar{c} A c} \rightarrow s^{1-(D / 2)} g_{\bar{c} A c}, \quad g_{A^{3}} \rightarrow s^{-1-(D / 2)} g_{A^{3}}, \quad g_{A^{4}} \rightarrow s^{-D} g_{A^{4}},
$$

so that all the coupling constants are perturbatively irrelevant [around the IR fixed point described by the action (9)], except for $g_{\bar{c} A c}$ at dimensions $D \leq 2$. In other words, $D=2$ is the upper critical dimension of the theory, in agreement with an analysis of the IR behavior of higher orders in perturbation theory [13]. Note that the scaling dimensions (12) are simply 
the mass dimensions of the coupling constants when one replaces the gluon field with the rescaled field $\tilde{A}_{\mu}^{a}$ according to Eq. (11).

The theory we will hence be considering in the following is defined by the free action (9) plus the sole interaction term

$$
S_{\mathrm{IR}}^{1}=\tilde{g} f^{a b c} \int d^{D} x \partial_{\mu} \bar{c}^{a} \tilde{A}_{\mu}^{b} c^{c}
$$

where we have redefined the coupling constant as $\tilde{g}=g_{\bar{c} A c} / m$. It will be interesting to consider this specific theory for dimensions $D \geq 2$, even though the only IR-stable fixed point for $D>2$ will turn out to be the trivial one (9), as expected. We will employ an epsilon expansion around $D=2$, i.e., we will consider dimensions $D=2+\epsilon$.

Note that neglecting the three- and four-gluon couplings (with the argument that these couplings are perturbatively irrelevant for any positive dimension) precisely corresponds to ghost dominance because then only the diagrams with the greatest number of ghost propagators remain. Ghost dominance can hence be considered as a consequence of the change in the scaling dimension of the gluon field which, in turn, is a consequence of the appearance of a gluon mass term.

For the RG analysis of the interacting theory we calculate the one-loop self-energies, first for the ghost fields. From the action (9) plus the interaction term (13) one obtains (suppressing the color factor)

$$
=-\frac{1}{2} \frac{N \bar{g}^{2}}{4 \pi} p^{2}\left(\frac{2}{\epsilon}+\gamma_{E}-\ln (4 \pi)+\ln \frac{p^{2}}{\kappa^{2}}\right)
$$

at $D=2+\epsilon$, where we have introduced a dimensionless coupling constant $\bar{g}$ via

$$
\tilde{g}^{2}=\left(\kappa^{2}\right)^{-\epsilon / 2} \bar{g}^{2}
$$

[cf. Eq. (12)]. For the gluon self-energy, on the other hand, one obtains in $D=2+\epsilon$ dimensions,

$$
m_{p}=\frac{1}{2} \frac{N \bar{g}^{2}}{4 \pi}\left[\left(\frac{2}{\epsilon}+\gamma_{E}-\ln (4 \pi)+\ln \frac{p^{2}}{\kappa^{2}}-2\right) \delta_{\mu \nu}+2 \frac{p_{\mu} p_{\nu}}{p^{2}}\right]
$$


Note that the gluon self-energy contains a longitudinal contribution which makes the breaking of BRST invariance as a result of ghost dominance explicit. In the following we only consider the transverse part of the self-energy (since the gluon propagator stays transverse) which is given by the coefficient of $\delta_{\mu \nu}$ in Eq. (16). We also emphasize that the counterterm for the self-energy (16) can be chosen local.

We now renormalize the theory in the standard way, rescaling the fields with field renormalization constants $Z_{A}$ and $Z_{c}$, and impose the following normalization conditions on the propagators of the renormalized fields:

$$
\begin{gathered}
\left.\left\langle\tilde{A}_{R, \rho}^{a}(p) \tilde{A}_{R, \sigma}^{b}(-q)\right\rangle\right|_{p^{2}=\mu^{2}}=\left.\frac{1}{Z_{A}}\left\langle\tilde{A}_{\rho}^{a}(p) \tilde{A}_{\sigma}^{b}(-q)\right\rangle\right|_{p^{2}=\mu^{2}}=\delta^{a b}\left(\delta_{\rho \sigma}-\frac{p_{\rho} p_{\sigma}}{p^{2}}\right)(2 \pi)^{D} \delta(p-q), \\
\left.\left\langle c_{R}^{a}(p) \bar{c}_{R}^{b}(-q)\right\rangle\right|_{p^{2}=\mu^{2}}=\frac{1}{Z_{c}}\left\langle\left. c^{a}(p) \bar{c}^{b}(-q)\right|_{p^{2}=\mu^{2}}=\frac{1}{\mu^{2}} \delta^{a b}(2 \pi)^{D} \delta(p-q) .\right.
\end{gathered}
$$

Upon substituting the expressions (14), (16) for the self-energies, the renormalization constants become functions of the renormalization scale $\mu$. The resulting one-loop anomalous dimensions read

$$
\begin{aligned}
& \gamma_{A}=\mu^{2} \frac{d}{d \mu^{2}} \ln Z_{A}=\frac{1}{2} \frac{N \bar{g}^{2}}{4 \pi}, \\
& \gamma_{c}=\mu^{2} \frac{d}{d \mu^{2}} \ln Z_{c}=-\frac{1}{2} \frac{N \bar{g}^{2}}{4 \pi} .
\end{aligned}
$$

The $\mu$-independence of the bare propagators implies, in general, that

$$
\begin{aligned}
\mu^{2} \frac{d}{d \mu^{2}}\left\langle\tilde{A}_{R, \rho}^{a}(p) \tilde{A}_{R, \sigma}^{b}(-q)\right\rangle & =-\gamma_{A}\left\langle\tilde{A}_{R, \rho}^{a}(p) \tilde{A}_{R, \sigma}^{b}(-q)\right\rangle, \\
\mu^{2} \frac{d}{d \mu^{2}}\left\langle c_{R}^{a}(p) \bar{c}_{R}^{b}(-q)\right\rangle & =-\gamma_{c}\left\langle c_{R}^{a}(p) \bar{c}_{R}^{b}(-q)\right\rangle .
\end{aligned}
$$

For the definition of the (dimensionless) renormalized coupling constant we consider the renormalized proper ghost-gluon vertex at the symmetric point,

$$
\begin{aligned}
\left.\Gamma_{R, \bar{c} \tilde{A} c}(p, q, r)\right|_{p^{2}=q^{2}=r^{2}=\mu^{2}} & =\left.Z_{A}^{1 / 2}(\mu) Z_{c}(\mu) \Gamma_{\bar{c} \tilde{A} c}(p, q, r)\right|_{p^{2}=q^{2}=r^{2}=\mu^{2}} \\
& =\mu^{-\epsilon / 2} \bar{g}_{R}(\mu) f^{a b c} i p_{\rho}(2 \pi)^{D} \delta(p+q+r) .
\end{aligned}
$$

(We have suppressed the Lorentz and color indices on the proper ghost-gluon vertex $\Gamma_{\bar{c} \tilde{A} c}$ for readability.) Simple power counting shows that the loop corrections to $\Gamma_{\bar{c} \tilde{A} c}$ at the symmetric point are $\mu$-independent (Taylor's theorem). Consequently, one finds for the beta function to one-loop order,

$$
\beta\left(\bar{g}_{R}\right)=\mu^{2} \frac{d}{d \mu^{2}} \bar{g}_{R}=\frac{1}{2} \bar{g}_{R}\left(\frac{\epsilon}{2}-\frac{1}{2} \frac{N \bar{g}_{R}^{2}}{4 \pi}\right) .
$$


For $\epsilon>0(D>2)$, we find a trivial IR-stable fixed point (as expected) and a nontrivial IR-unstable fixed point. Although the latter is irrelevant for the IR physics, for the sake of comparison with the results of the DS analysis it is interesting to determine the IR behavior of the propagators corresponding to this fixed point. Substituting the unstable fixed-point value of $\bar{g}_{R}$ in Eq. (18) and integrating the differential equations (19) for the renormalized propagators with these fixed point values for the anomalous dimensions and the normalization conditions (17) as initial conditions yields

$$
\begin{aligned}
\left\langle\tilde{A}_{R, \rho}^{a}(p) \tilde{A}_{R, \sigma}^{b}(-q)\right\rangle & =\left(\frac{p^{2}}{\mu^{2}}\right)^{\epsilon / 2} \delta^{a b}\left(\delta_{\rho \sigma}-\frac{p_{\rho} p_{\sigma}}{p^{2}}\right)(2 \pi)^{D} \delta(p-q), \\
\left\langle c_{R}^{a}(p) \bar{c}_{R}^{b}(-q)\right\rangle & =\frac{1}{p^{2}}\left(\frac{\mu^{2}}{p^{2}}\right)^{\epsilon / 2} \delta^{a b}(2 \pi)^{D} \delta(p-q) .
\end{aligned}
$$

The IR-limit of the propagators is hence of the form (21), and the exponents coincide exactly with the scaling solution (4) of the DS equations found in Refs. [2, 3].

The other fixed point in Eq. (21), which is IR-stable and hence physically relevant for the description of the IR regime of the theory in $D>2$ dimensions, is trivial in accord with our identification of $D=2$ as the upper critical dimension. Apart from a vanishing renormalized coupling constant, one hence finds the "classical" behavior of the propagators in the IR limit, which means in particular that (the transverse part of) the gluon propagator tends toward a non-vanishing constant for zero momentum [cf. Eq. (9)]. The trivial fixed point then corresponds to the decoupling solution [5-7] of the DS equations described in the paragraph after Eq. (6). The triviality of the fixed point justifies the use of perturbation theory for the decoupling solution [13].

We can obtain a more precise description of the IR behavior of the propagators and the renormalized coupling constant by analyzing the approach to the (stable) IR fixed point as the momentum scale tends to zero. Namely, integrating the differential equation (21) for $\bar{g}_{R}(\mu)$ gives

$$
\frac{N \bar{g}_{R}^{2}(\mu)}{4 \pi}=\frac{\left(\mu^{2} / \Lambda^{2}\right)^{\epsilon / 2}}{1+\left(\mu^{2} / \Lambda^{2}\right)^{\epsilon / 2}} \epsilon,
$$

where $\Lambda$ is the (undetermined) scale with

$$
\frac{N \bar{g}_{R}^{2}(\Lambda)}{4 \pi}=\frac{\epsilon}{2},
$$

"halfway" between the two fixed points. Substituting the expression for $\bar{g}_{R}(\mu)$ from Eq. (23) for $\bar{g}$ in the anomalous dimensions (18) and using the result in the integration of the 
differential equations (19) for the renormalized propagators [together with Eq. (17)] leads to

$$
\begin{aligned}
\left\langle\tilde{A}_{R, \rho}^{a}(p) \tilde{A}_{R, \sigma}^{b}(-q)\right\rangle & =\frac{1+\left(p^{2} / \Lambda^{2}\right)^{\epsilon / 2}}{1+\left(\mu^{2} / \Lambda^{2}\right)^{\epsilon / 2}} \delta^{a b}\left(\delta_{\rho \sigma}-\frac{p_{\rho} p_{\sigma}}{p^{2}}\right)(2 \pi)^{D} \delta(p-q), \\
\left\langle c_{R}^{a}(p) \bar{c}_{R}^{b}(-q)\right\rangle & =\frac{1}{p^{2}} \frac{1+\left(\mu^{2} / \Lambda^{2}\right)^{\epsilon / 2}}{1+\left(p^{2} / \Lambda^{2}\right)^{\epsilon / 2}} \delta^{a b}(2 \pi)^{D} \delta(p-q) .
\end{aligned}
$$

Also note that, from Eq. ([6) $g_{R}^{2}\left(p^{2}\right)=\bar{g}_{R}^{2}\left(\mu^{2}=p^{2}\right.$ ) (except for a constant factor from the finite renormalization of the ghost-gluon vertex).

The result (25) for the propagators is at least qualitatively confirmed by calculations on very large lattices [8-10]. In dimension $D=3$, the linear rise of the gluon propagator with $|p|$ from a constant finite value in the IR is clearly seen [17] (incidentally, this is very different from the usual behavior of a massive propagator). In $D=4$ dimensions, a (approximately) quadratic rise of the gluon propagator with $|p|$ in the IR as predicted by Eq. (25) is found in lattice simulations with lattice parameter $\beta=0$, i.e., without any contribution from the original Yang-Mills action [18]. In order to compare our results in four dimensions to the case of finite $\beta$, we have to reintroduce the term $\left(A_{\mu}^{a} p^{2} A_{\mu}^{a}\right)$ in the action, since the latter term is of the same order in $\left(p^{2} / m^{2}\right)$ as the correction to the "classical" behavior generated by the application of the RG (at $\epsilon=2$ ). Given that there are no loop corrections to the $\left(A_{\mu}^{a} p^{2} A_{\mu}^{a}\right)$-term at one-loop order, the resulting gluon propagator is precisely of the form obtained in the refined Gribov-Zwanziger scenario [15].

We also mention that a semi-quantitative reproduction of the lattice results (for the decoupling solution) over the whole momentum range was achieved in $D=3$ and 4 dimensions in one of the renormalization schemes proposed in Ref. [13]. The scheme in question implements normalization conditions different from (17) for the theory defined by the full action (7). No epsilon expansion is employed in this approach, and the only IR fixed point of the coupling constant found in four dimensions is stable and trivial, while the three-dimensional IR-stable fixed point is nontrivial.

In summary, our RG analysis recovers, in dimensions $D>2$, the decoupling solution and one of the scaling solutions [given in Eq. (44)] of the DS equations. Moreover, unlike the DS equations, the RG analysis is able to show that the decoupling solution is IR-stable and hence physically relevant, while the scaling solution (41) is IR-unstable. In dimension $D=2$, the beta function (21) is negative for $\bar{g}_{R} \neq 0$, hence the only fixed point, the trivial one corresponding to the decoupling solution, becomes IR-unstable. This is consistent with 
lattice calculations which do not find decoupling (but rather scaling) behavior in the IR at $D=2[11]$.

What about the other scaling solution of the DS equations given in Eq. (5))? As we shall now demonstrate, it can also be obtained from the RG analysis if the so-called horizon condition [14] of the original (not refined) Gribov-Zwanziger scenario,

$$
F\left(p^{2}\right) \rightarrow \infty \text { for } \quad p^{2} \rightarrow 0
$$

in the notation of Eq. (1), is implemented as a normalization condition. The horizon condition is fulfilled for the scaling solutions (44) and (5), but not for the decoupling solution. Note that we have obtained the scaling solution (4) from the RG analysis without ever using the horizon condition explicitly.

Now, in order to implement Eq. (26) classically, we change the free part (9) of the IR-form of the action to

$$
S_{\mathrm{IR}}^{0}=\int d^{D} x\left(\frac{1}{2} A_{\mu}^{a} m^{2} A_{\mu}^{a}+\frac{1}{b^{2}} \partial_{\mu} \bar{c}^{a}\left(-\partial^{2}\right) \partial_{\mu} c^{a}+i B^{a} \partial_{\mu} A_{\mu}^{a}\right),
$$

where the parameter $b$ with the dimension of mass is introduced in order to maintain the usual canonical dimensions of the ghost fields. The form of the ghost term in Eq. (27) is known as a (isotropic) Lifshitz point in statistical physics [19, 20].

It is clear from Eq. (27) that the scaling dimensions of the ghost fields differ from the usual canonical dimensions, namely, the invariance of the free action under the rescaling $x \rightarrow x / s$ implies the field rescalings

$$
c^{a}(x) \rightarrow s^{(D / 2)-2} c^{a}(s x), \quad \bar{c}^{a}(x) \rightarrow s^{(D / 2)-2} \bar{c}^{a}(s x) .
$$

As for the coupling constants, the three- and four-gluon couplings scale as before in Eq. (12), since the scaling of the gluon field is unchanged with respect to Eq. (10), while for the ghost-gluon coupling we find

$$
g_{\bar{c} A c} \rightarrow s^{3-(D / 2)} g_{\bar{c} A c} .
$$

As a consequence, the upper critical dimension of the theory is now $D=6$. We rewrite the sole interaction term (13) as

$$
S_{\mathrm{IR}}^{1}=\tilde{g} f^{a b c} \int d^{D} x \frac{1}{b^{2}} \partial_{\mu} \bar{c}^{a} \tilde{A}_{\mu}^{b} c^{c},
$$

where we have defined a new coupling constant $\tilde{g}$ as $\tilde{g}=\left(b^{2} / m\right) g_{\bar{c} A c}$. 
Following in strict analogy the procedure applied before to the action given by Eqs. (9) and (13) (details will be provided elsewhere), we again find two fixed points, only that this time the trivial fixed point is IR-unstable and the nontrivial fixed point is IR-stable in dimensions $D<6$. Plugging the value for the stable fixed point into the anomalous dimensions yields a scaling solution with IR exponents [in the notation of Eq. (2)]

$$
\alpha_{F}(D)=\frac{5 D-6}{24}, \quad \alpha_{G}(D)=-\frac{18-D}{12}
$$

Comparing the result (31) to (the approximation to) the solution (5) of the DS equations

obtained in Refs. [2, 3], we find that both coincide (exactly) at $D=6$, and the difference between them in the range $2 \leq D \leq 4$ is largest for $D=2$, where $\alpha_{F}=1 / 5$ (exactly) from the solution of the DS equation and $\alpha_{F}=1 / 6$ from Eq. (31). However, considering the usually slow convergence (after Borel resummation) of the epsilon expansion in applications to statistical physics, the coincidence of Eq. (31) with the solution (15) of the DS equations is surprisingly good.

Together with the previous dicussion, we now have found two different IR-stable fixed points. Which one is physically realized? The realization of the fixed point corresponding to the IR exponents (31) depends on the fine-tuning of the ghost term appearing in the action (9), in order to fulfill the horizon condition. The latter term is perturbatively relevant with the scaling dimensions (28) of the ghost fields. Hence, unless there is a physical reason for the fine-tuning of this term or the imposition of the horizon condition (as there actually has formerly been thought to be), the fixed point is unstable with respect to perturbations of the term in question.

In summary, the RG analysis also reproduces the second scaling solution (5) upon implementing the horizon condition. However, this condition is IR-unstable under the RG flow, hence in the end the only IR-stable fixed point is the one corresponding to the decoupling solution (in dimensions $D>2$ ), in agreement with recent lattice simulations.

\section{Acknowledgments}

The author would like to thank David Dudal, José Rodríguez-Quintero, and Chris Stephens for valuable discussions, and David Dudal and Chris Stephens for a critical reading of the manuscript and helpful comments. Support by CIC-UMSNH and Conacyt project 
CB-2009/131787 is gratefully acknowledged.

[1] L. von Smekal, A. Hauck, and R. Alkofer, Phys. Rev. Lett. 79, 3591 (1997); Ann. Phys. (N.Y.) 267, 1 (1998).

[2] D. Zwanziger, Phys. Rev. D 65, 094039 (2002).

[3] C. Lerche and L. von Smekal, Phys. Rev. D 65, 125006 (2002).

[4] J. C. Taylor, Nucl. Phys. B33, 436 (1971).

[5] A. C. Aguilar and A. A. Natale, JHEP 0408, 057 (2004).

[6] P. Boucaud, T. Brüntjen, J. P. Leroy, A. Le Yaouanc, A. Lokhov, J. Micheli, O. Pène, and J. Rodríguez-Quintero, JHEP 0606, 001 (2006).

[7] M. Frasca, Phys. Lett. B670, 73 (2008).

[8] I. L. Bogolubsky, E.-M. Ilgenfritz, M. Müller-Preussker, and A. Sternbeck, Proc. Sci., LAT2007 (2007) 290.

[9] A. Cucchieri and T. Mendes, Proc. Sci., LAT2007 (2007) 297.

[10] A. Sternbeck, L. von Smekal, D. B. Leinweber, and A. G. Williams, Proc. Sci., LAT2007 (2007) 340 .

[11] A. Maas, Phys. Rev. D 75, 116004 (2007).

[12] G. Curci and R. Ferrari, Nuovo Cim. A 32, 151 (1976).

[13] M. Tissier and N. Wschebor, Phys. Rev. D 82, 101701 (2010); ibid. 84, 045018 (2011).

[14] D. Zwanziger, Nucl. Phys. B399, 477 (1993).

[15] D. Dudal, J. A. Gracey, S. P. Sorella, N. Vandersickel, and H. Verschelde, Phys. Rev. D 78, 065047 (2008).

[16] M. Le Bellac, Quantum and Statistical Field Theory, Oxford University Press 1991.

[17] A. Cucchieri and T. Mendes, Proc. Sci., QCD-TNT09 (2009) 026.

[18] A. Cucchieri and T. Mendes, Phys. Rev. D 81, 016005 (2010).

[19] R. M. Hornreich, M. Luban, and S. Shtrikman, Phys. Rev. Lett. 35, 1678 (1975).

[20] C. Mergulhão, Jr. and C. E. I. Carneiro, Phys. Rev. B 58, 6047 (1998). 